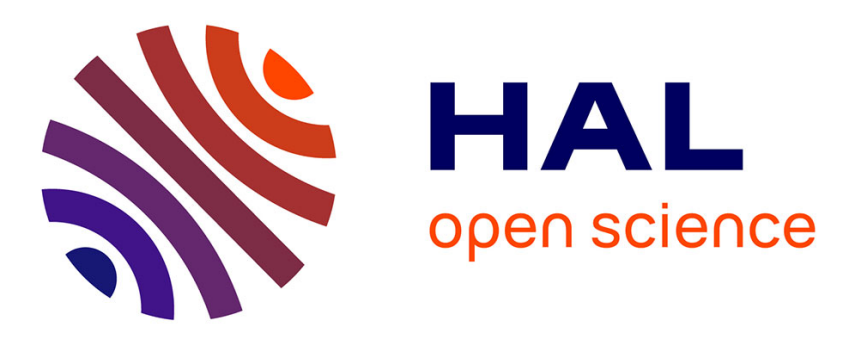

\title{
A communication stack over PLC for multi physical layer IPv6 Networking
}

Cedric Chauvenet, Bernard Tourancheau, Denis Genon-Catalot, Pierre-Emmanuel Goudet, Mathieu Pouillot

\section{To cite this version:}

Cedric Chauvenet, Bernard Tourancheau, Denis Genon-Catalot, Pierre-Emmanuel Goudet, Mathieu Pouillot. A communication stack over PLC for multi physical layer IPv6 Networking. SmartGridCom, 2010, Washington, United States. inria-00563226

\section{HAL Id: inria-00563226 \\ https://hal.inria.fr/inria-00563226}

Submitted on 4 Feb 2011

HAL is a multi-disciplinary open access archive for the deposit and dissemination of scientific research documents, whether they are published or not. The documents may come from teaching and research institutions in France or abroad, or from public or private research centers.
L'archive ouverte pluridisciplinaire HAL, est destinée au dépôt et à la diffusion de documents scientifiques de niveau recherche, publiés ou non, émanant des établissements d'enseignement et de recherche français ou étrangers, des laboratoires publics ou privés. 


\title{
A communication stack over PLC for multi physical layer IPv6 Networking
}

\author{
Cedric Chauvenet: Watteco Inc. and CITI Insa-Lyon / INRIA, France \\ Bernard Tourancheau: CITI Insa-Lyon / INRIA and LIP UMR 5668 CNRS-ENS-INRIA-Université Lyon1, France \\ Denis Genon-Catalot: LCIS EA 3747 GrenobleINP-Université Grenoble2, France \\ Pierre-Emmanuel Goudet, Mathieu Pouillot: Watteco Inc., France \\ Email: c.chauvenet@watteco.com, Bernard.Tourancheau@ens-lyon.fr, Denis.Genon-Catalot@iut-valence.fr
}

\begin{abstract}
In this paper, we propose a communication stack for powerline communication (PLC) based on open standards. Our aim is to provide interoperability features regarding others media with a robust and reliable communication stack for smart metering, home control or home area network applications. Our work is based on the adaptation of the IEEE 802.15.4 standard protocol over PLC. It is constrained by the low-power, lossy and low data-rate context of powerline transceiver that uses pulse modulation. We first make a quick survey and justify the use of the IEEE 802.15.4 standard over PLC by drawing a parallel with Low-Rate Wireless Personal Area Networks (LR-WPAN). We then focus on the convergence of the IPv6 protocol at the network level, with the 6LoWPAN adaptation. We present our initial implementation of the RPL setup and routing protocol. This allows for a full network layer stack and results in efficient routing in our low power, low data-rate and lossy network context. We then present a real testbed of this stack and the step by step validation of its performance with experiments of data exchanges between PLC nodes. Finally we present interoperability tests performed between wireless and PLC nodes. We conclude about the interest of such interoperability for the real usage of sensor networks with a feedback from field's applications deployment and our future work.
\end{abstract}

\section{INTRODUCTION}

We are now dealing with the Internet of things, that focuses on everything that could be connected, but is currently not. In this context there is definitively a need for heterogeneity, and the use of open protocols, to enable the cohabitation between networks and create a real interconnectivity through all these devices.

We assume that a single medium technology cannot fulfill every requirement, and that there will be always a need for the use of different technologies together to cover all the needs that will appear in the future. With this assumption, we present in the following a way to provide a reliable and robust interoperability in a low power and lossy networks (LLN) context. In particular we focus here on low rate wireless personal area networks (LR-WPAN), for which the IEEE 802.15.4 standard [1] has been designed. The vast majority of these devices are wireless and we brings here an easy interoperability feature on another medium using Powerline Communication (PLC).

\section{A. Wireless medium in LR-WPAN}

The wireless devices using the IEEE 802.15.4 standard provide good mobility features, roughly limited by the zone of reachability of at least one of their neighbors. The principal limitation of these devices is the management of their power resource that is limited to the battery capacity or energy scavenging. The management of the energy available is really a key point for these devices, and is directly linked to the lifetime of the whole network. Many works aims to optimize the network lifetime by optimizing the power management with the use of duty cycling (in various MAC protocols such as S-MAC[2], T-MAC[3], or Z-MAC [4]), efficient algorithm design [5] or sink positioning [6].

\section{B. PLC medium in LR-PAN}

The powerline medium can't provide the same mobility feature but the power resource management is not a problem, because the device can directly access the main grid power supply. However, power consumption is still relevant to keep the global power overhead very low when considering numerous devices. In PLC world, different coupling technologies exist that provide different networking characteristics such as data rates, transmission range, error rate with different needs in power load and different perturbation on the power line. We study in this paper a low cost, low power and low rate technology very suitable for sensor networking on LR-PAN. The range provided by this PLC technology can reach up to $1 \mathrm{~km}$ in a urban context, regardless on the environment, but is depending on the electrical activity on the grid.

\section{CONTEXT}

The devices considered here are low data-rate, low power and use a lossy medium. The expectation with these devices is to provide a simple and reliable connectivity to any objects for their low data-rate applications. The power consumption of LR-PAN devices has to be low, because they are often on battery or numerous and they should not create a power consumption overload on the grid. The data rate is low because it is related to power consumption. Moreover, typical applications such as smart metering, home control or home area networks applications do not require large throughput. A few tens of $\mathrm{kb} / \mathrm{s}$ is enough. In a wireless medium, others wireless devices or obstacles can disturb the communication. Similarly, in a powerline medium, electrical devices can be plugged/unplugged or switch on/off so that it changes the 
behavior of the medium. We can not avoid these perturbations due to real-life activity, and the communication has to adapt to them. Finally, these devices have limited memory and processing capabilities due to their low consumption, integration requirement and cost constraints. This constrained context needs a specific communication protocol, such has the design of the IEEE 802.15.4 standard.

\section{PHY AND MAC LAYER : IEEE 802.15.4}

This standard defines the physical layer (PHY) and medium access control (MAC) sublayer specifications for low data rate wireless connectivity with very limited power consumption requirements typically operating in the personal operating space (POS) of $10 \mathrm{~m} \mathrm{[1].} \mathrm{The} \mathrm{frame} \mathrm{length} \mathrm{is} \mathrm{limited} \mathrm{to} 128$ bytes to ensure reasonably low packet error rates as bit-error rates are non-negligible in our lossy context. The medium access control defined in the standard is the carrier sense multiple access with a collision avoidance (CSMA/CA).

Even if the scope of the standard is for wireless devices, we first investigated the idea of using it over raw PLC in [7]. Indeed we found that the definition of a LR-WPAN in the 802.15.4 standard is very close to our PLC testbed context ${ }^{1}$.

The PLC nodes we consider have roughly the same architecture as classic RF 802.15.4 nodes. The processing is ensured by a micro-controller ${ }^{2}$, and the communication is managed by a PLC transceiver which roughly emulates a radio transceiver on the SPI interface. The PHY and a major part of the MAC (MAC acknowledgments and CSMA/CA) is implemented in this PLC transceiver. The micro-controller is managing the compliance with the 802.15.4 frame format and the upper layer of the communication stack. However this transceiver provides a $10 \mathrm{~kb} / \mathrm{s}$ data-rate that induces some adaptation within the MAC part. This adaptation provides a communication on powerline with the 802.15.4 data format. Moreover, this transceiver aims to keep its power consumption $(10 \mathrm{~mW})$, footprint $\left(25 \mathrm{~mm}^{2}\right)$ and cost (\$ order) as low as possible in order to be easily integrable. Finally, the scope of this standard is tight to our context and most of the topic addressed by this standard are suitable for PLC. In particular, PLC is not a broadcast domain like ethernet because of its noisy, lossy and variable behavior, and 802.15.4 brings an efficient solution to such a medium.

Our implementation of the 802.15.4 standard over PLC is based on the 2003 revision. This implementation allow us to send PLC frames of 128 bytes maximum, which is very suitable for a lossy links such as PLC. This implementation also handles the CSMA-CA protocol which provide a suitable solution for collision avoidance. The major difference with CSMA-CA over RF is that our PLC implementation uses the $50 \mathrm{~Hz}$ voltage as a backoff period. This is due to the transmission capability of the PLC transceiver which is limited to a window around the rising zero crossing voltage. Further investigation about MAC layer consideration over PLC is under way.

\footnotetext{
${ }^{1}$ The "PLC transceiver" is called $\mathrm{WPC}^{\mathrm{TM}}$ for Watt Pulse Communication because of its specific coupling method, see Watteco Inc. www.watteco.com

${ }^{2} \mathrm{An}$ ATmega1281 is used in the Watteco devices.
}

\section{NETWORK LAYER}

\section{A. IPv6}

To overcome the declining unallocated address space and in anticipation that networked appliances and instruments will outnumber conventional computer hosts, IPv6 extends the IP address space from 32 to 128 bits and directly addresses very important issues such as auto-configuration, security, multicasting, .... This is especially welcome in the growing Internet of things we are speaking of. Recognizing the growth in link bandwidth in the wired Internet, IPv6 also increases the minimum MTU requirement from 576 to 1,280 bytes.

IP protocols are defined by the Internet Engineering Task Force (IETF) in the form of Request For Comments open standard protocols (RFC). These specifications provides a complete service for IPv6 networks and they were recently proven adaptable and operational for networking on small devices [8], [9], [10]. The IETF working groups about sensor activities started from these significant developments, by defining a way to carry the IPv6 MTU into smaller frames with RFC4944, security in RFC4301, auto-configuration in RFC4862 and IETF drafts for routing and other aspects of the protocols.

As presented in [11], an IPv6 stack can be implemented in approximately $11.5 \mathrm{kB}$ of ROM and $1.8 \mathrm{kB}$ of RAM. With a complete run-time (timers, scheduler, etc.) as well as RFCcompliant UDP and TCP protocols above, an OS that provides a complete IPv6 network stack can be implemented within 35 $\mathrm{kB}$ of ROM and $3 \mathrm{kB}$ of RAM. [10] pull down the memory requirement to $24 \mathrm{kB}$ of $\mathrm{ROM}$ and $3.6 \mathrm{kB}$ of RAM. So complete IPv6-based applications can fit in a micro-controller providing only $64 \mathrm{kB}$ of ROM and 4 to $8 \mathrm{kB}$ of RAM which is the order of magnitude of today's micro-controller such as the PLC nodes we consider.

As presented in Section 6, the footprint of our IPv6 stack is $16.444 \mathrm{kB}$ of ROM and $2.622 \mathrm{kB}$ of RAM. This include main IPv6 functionalities such as IPv6 Specification (RFC2460), IPv6 Neighbor Discovery (RFC4861), ICMPv6 (RFC4443) and Stateless Address Auto-configuration (RFC4862). As a result it brings to our PLC devices a complete and lightweight IPv6 connectivity. Optimization of some of these protocols for LLNs have been designed in the IETF 6LoWPAN working group.

\section{B. 6LoWPAN}

With RFC4944, the IETF has defined the 6LoWPAN adaptation layer that includes a compression mechanism of the IPv6 header (now described in the 6lowpan-HC draft).This mechanism is stateless which means that it creates no binding state between the compressor/decompressor pair. Most of link technologies designed for smart objects do not support the full 1280-byte MTU that IPv6 require. For instance, IEEE 802.15.4 only supports 127 -byte MTUs so a full IPv6 packet do not fit in an IEEE 802.15.4 frame.

6LoWPAN provides header compression to reduce transmission overhead, fragmentation to support the IPv6 minimum MTU requirement, and support for layer-two forwarding to deliver IPv6 datagram over multiple hops. 
802.15.4 protocol data units have different sizes depending on how much overhead is present [1]. Starting from a maximum physical layer packet size of 127 bytes (aMaxPHYPacketSize) and a maximum frame overhead of 25 (aMaxFrameOverhead), the resultant maximum frame size at the media access control layer is 102 bytes. Link-layer security imposes further overhead, which in the maximum case ( 21 bytes of overhead in the AES-CCM-128 case, versus 9 and 13 for AES-CCM-32 and AES-CCM-64, respectively) leaves only 81 bytes available. This is obviously far below the minimum IPv6 packet size of 1280 bytes (RFC2460). Thus a fragmentation and reassembly adaptation layer must be provided at the layer below IP. Furthermore, since the IPv6 header is 40 bytes long, this leaves only 41 bytes for upperlayer protocols, like UDP. The latter uses 8 bytes in the header which leaves only 33 bytes for application data fragmentation encapsulation.

The memory footprint of our 6LoWPAN header compression mechanism is $4.922 \mathrm{kB}$ of ROM and 291 Bytes of RAM. This implementation is based on the HC-06 draft. As a result, it reduces IPv6 overhead and enhances our PLC performances. 6LoWPAN effect on PLC performance is presented in the experimentation section.

\section{ROUTING}

The 6LoWPAN adaptation layer brings all the IPv6 mechanisms needed for large scale deployments and easy interoperability available for LR-WPAN with a minimum overhead. But these new IPv6-compliant nodes are very different from classic networking devices and routing over low power and lossy networks introduces requirements that existing routing protocols may not fully address.

Limited memory and communication capabilities constrain the routing state at each node as well as the routing information that might be communicated. These restrictions forbid the use of protocols that rely on complete link-state information. Traditional distance vector mobile ad-hoc networks (MANet) protocols are also unsuitable because most of them exchange route maintenance information at rates that exceed typical LR-WPAN communication and react to unreachability with expensive route-repair mechanisms. Instead, LoWPAN routing protocols must operate with incomplete information and tolerate some inconsistency.

The draft from P.Levis ${ }^{3}$ provides a brief survey of the strengths and weaknesses of existing protocols and examines whether existing and mature IETF protocols can be used without modification in these networks, or whether further work is necessary. It concludes that no existing IETF protocol meets the requirements of this domain, as existing protocols were not designed with all of the constraints of our context. They have made trade-offs which may or may not be appropriate for Low power and Lossy Networks (LLNs).

To achieve the design of this new routing protocol, a new IETF working group called Routing Over Low-power and Lossy networks (ROLL ${ }^{4}$ ) was formed in 2008. This group has

\footnotetext{
${ }^{3}$ draft-ietf-roll-protocols-survey-07

${ }^{4}$ http://tools.ietf.org/wg/roll/
}

designed the IPv6 Routing Protocol for Low power and Lossy Networks (RPL) ${ }^{5}$ that should be able to operate over a variety of different link layers, including but not limited to low power wireless or PLC technologies. Note that there is no "wireless" word in the name of protocol, because it does not rely on any particular features of a specific link layer technology. These features brings flexibility to the RPL protocol. This fits with our goal of providing interoperability in LLNs.

The ROLL working group has designed several drafts such as draft-ietf-roll-building-routing-reqs (RFC 5867) and draftietf-roll-home-routing-reqs (RFC 5826), tightening the use of the RPL protocol in the application field of our PLC devices.

Figure 1 show an example of ROLL architecture. RPL enable Multipoint-to-Point (MP2P), Point-to-multipoint (P2MP) and a basic structure for point-to-point (P2P) traffic. The protocol model of RPL is based on the construction and the maintenance of one or several Directed Acyclic Graphs (DAGs). To achieve this, RPL defines DIO,DIS and DAO, three types of a new ICMPv6 message called RPL Control Message.

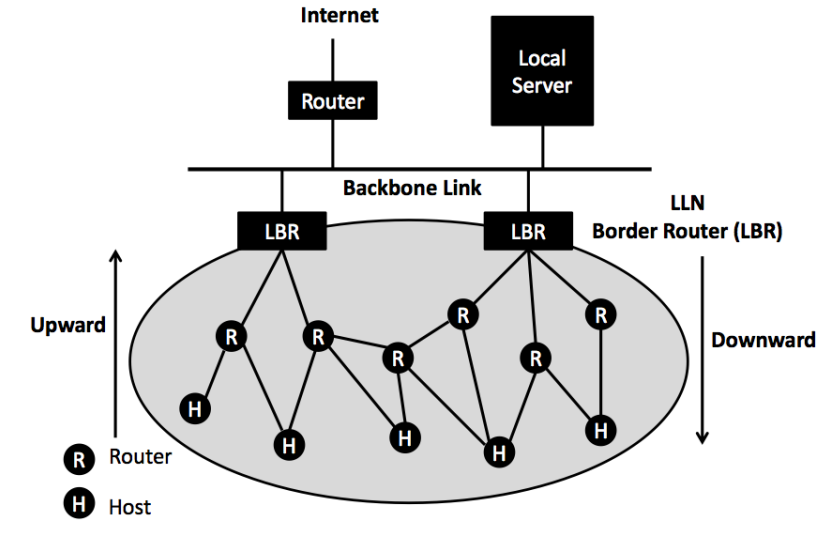

Figure 1. The ROLL architecture

The RPL protocol is well suited for our PLC nodes for several reasons : This is a routing protocol specifically designed for LLNs, which is precisely our context. Indeed, it considers a set of constraints that were not in the motivation of other routing protocols but are of great importance in LLNs. This protocol is not related to a particular physical interface, so it is compliant with our PLC medium, and enable also to be used over others medium to create heterogeneous networks. The RPL protocol enable efficient MP2P traffic which is the principal data flows in smart-grid application. For example, all nodes will report their electricity, gas or water consumption at periodic times. RPL also provides efficient P2MP traffic which can be useful in the same smart-grid context to inform all nodes about power pricing informations and optimizing the energy consumption. It is also useful for street lighting application where a master will command all the lights from the street. RPL employs the trickle timer technique [12] that adapts the management traffic to the stability of the networks, and avoid greedy traffic to manage the network. RPL also

${ }^{5}$ http://tools.ietf.org/wg/roll/draft-ietf-roll-rpl/ 
allows mesh topology which increase our reachability capacity, and decrease the risks of unreachability zones on the power grid. The objective function concept offers a very wide range of applications, dealing with delay, power consumption or other metrics constraints independently from the physical layer used. Finally, RPL rely on the wide used IPv6 protocol which provide an easy connection with many web applications. The security aspect is currently in progress in ROLL, and it is a also an important feature needed for our applications.

Our RPL implementation has a memory footprint of 7.057 $\mathrm{kB}$ of ROM and 442 Bytes of RAM. A routing table entry takes 46 Bytes of RAM, and a neighbor table entry needs 44 Bytes of RAM.To fit with our micro controller RAM capacity, we limited our RPL implementation to 15 Neighbors, and 15 routing entries per node.

\section{EXPERIMENTATION}

We describe here how we implement a stack following the previously described characteristics on our PLC testbed, and show a real interoperability testbed.

\section{A. Hardware}

1) WPCDevKit: This device acts as PLC nodes. It is composed of 4 major parts : A PLC transceiver, a microcontroller, a USB interface and an alimentation. The PLC transceiver is a $\mathrm{WPC}^{\mathrm{TM}}$ from Watteco, the micro-controller is an ATmega 1281 and drive the transceiver through an SPI interface. The USB is used as a serial interface to provide some debugs and act on the node. The alimentation module create a $5 \mathrm{~V}$ power supply from the $110-220 \mathrm{AC}$ voltage input. The USB part is opto-isolated from the high voltage part.

2) Ethernet to PLC gateway: To provide the connection with our local PLC network, we developed a PLC to Ethernet gateway based on the Atmel RZUSBSTICK architecture. This device create an ethernet over USB emulation. It is based on an AT90USB1287 micro-controller which drive the $\mathrm{WPC}^{\mathrm{TM}}$ transceiver the same way as the ATmega1281. This micro-controller is integrated on a separate PCB so that it can be plugged on a classic WPCDevKit. It avoids to recreate a whole PLC node. This card directly integrates the USB interface with high speed isolators. With this card on the WPCDevKit, it allows a PC or a router too see our PLC node as an ethernet interface. Our PLC network is therefore seen as a subnet from the PC or the router connected.

This ethernet emulation as 2 main contributions: It creates a link with other networking devices, and it can be used as a sniffer on Wireshark ${ }^{6}$. The second capability is a major achievement because it enables the use of a performant network analyzer on PLC medium.

3) Low power development platform: To test our implementation, we used a low power PLC platform from Watteco. This platform brings the ability of manipulating safely (without the risk of high voltage) and reduces the medium as a short and simple wire to put away the possible perturbations of a real powerline medium in order to focus specifically on

\footnotetext{
${ }^{6} \mathrm{http} / / /$ www.wireshark.org
}

the stack performance. As shown in figure 2, our platform is made of a $220 \mathrm{~V} / 24 \mathrm{~V}$ AC converter, $7 \mathrm{WPCDevKit}$ and a 7 ports hub. Each PLC node of the platform has a strictly identical architecture as the WPCDevKit from Watteco. The $\mathrm{WPC}^{\mathrm{TM}}$ transceiver of each PLC node is driven by an ATmega 1281 or AT90USB1287 micro-controller, depending on the function intended. A connexion between all PLC nodes supply the power and provide the powerline communication medium (on $24 \mathrm{~V}$ here). All nodes have a USB port to provide a serial interface. We use this interface to act on each node (to control the topology of the network for example) and bring back some debug informations.

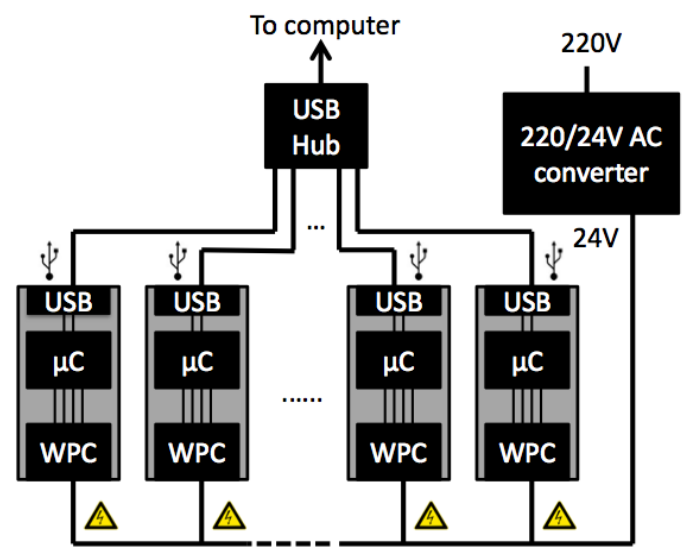

Figure 2. Low voltage PLC Platform

We first use this platform alone to create an homogeneous network on PLC. This platform will be connected to a RF network through a router in the interoperability experimentation.

\section{B. Software}

1) PLC node: Our stack is running under Contiki [13] in its 2.4 version ${ }^{7}$. We use the uIP IPv6 stack from Contiki which implements a full IPv6 stack. We have created a specific PLC platform in Contiki 2.4. Because our transceiver is roughly a RF transceiver emulation, the major part of the adaptation on Contiki is in the MAC layer and the uIP stack don't need any modifications. Our implementation of RPL is the result of a collaborative work with SICS. We have implemented the HC06 draft ${ }^{8}$ of 6LoWPAN and the version 5 of the RPL draft 9 . To performs our tests, we used the UDP sender and UDP client example available in the Contiki OS.

2) PLC gateway: There are 2 different softwares for this gateway. The first one creates an Ethernet emulation over USB and is derived from the RZUSBSTICK platform available in Contiki The second one is used as a sniffer application and has been developed in the $15 \operatorname{dot} 4$ tools project ${ }^{10}$ managed by Colin O'Flynn. Though this project is related to classic 15.4 RF nodes, the RF transceiver emulation of our specific PLC transceiver allow us to use this software over powerline.

\footnotetext{
${ }^{7}$ http://www.sics.se/contiki/

${ }^{8}$ http://tools.ietf.org/html/draft-ietf-6lowpan-hc-06

${ }^{9}$ http://tools.ietf.org/html/draft-ietf-roll-rpl-05

${ }^{10} \mathrm{http}: / /$ sourceforge.net/projects/dot4-tools/
} 
3) PLC networking: We have first created a RPL network on our PLC platform. We used a node with an ethernet emulation as a sniffer with the Wireshark software. When we first plug a PLC node, it declared itself as a root because it get no response to its DIS RPL control message. When we connect other nodes, they received a DIO response from the root to their DIS request and declared themselves as leaf attached to the DAG. Through the serial interface on each node, we can send some commands and forces a node to drop the packet sent by another node. With these command, we can block the communication between a node and the root, to detach this node from the root. When the node send its DIS, it will only receive DIO from nodes and will associate to a node. As a result, this node will associate to the DAG with a higher rank than other nodes. This is a simple way for manually controlling the topology of the network and reproduce unreachability that can appear in real experiments. When the DAG is settled, we checked successfully the trickle mechanism on the DIO sending, with the doubling of the time after each expiration. Our DAG construction is based on the Expected transmission count (ETX) metric. This metric compute the node rank with the success ratio of transmissions over a link. This metric is one of the suggested in the draft-ietf-roll-routing-metrics document.

4) PLC performances: Based on the previous description, we performed a testbed to show the benefits from the stacks implemented over PLC. Based on a simple topology created and maintain with RPL, we performed some pings series to highlight the 6LoWPAN header compression benefits. Results are plotted in figure 3 and provide a rough order of magnitude of PLC performance. To show the 6LoWPAN benefit over

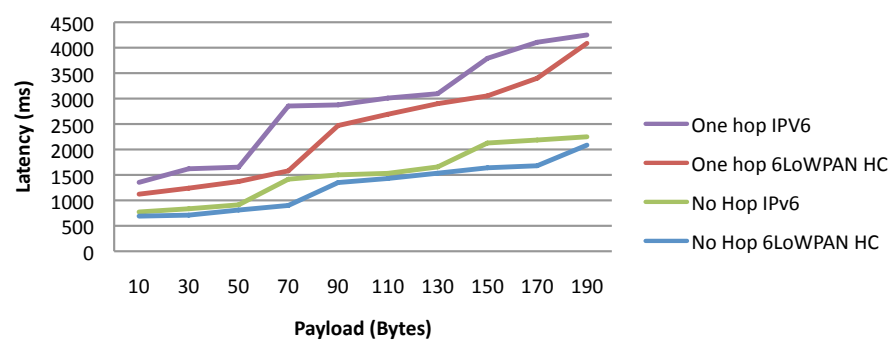

Figure 3. 6LoWPAN HC effect on PLC latency

PLC, we made successive pings and show the average latency when increasing the payload in the ping message. This payload correspond to the payload of the ICMPv6 message. First observation in figure 3 is that our minimal round trip latency for ping is around $700 \mathrm{~ms}$. This illustrates well the data rate difference with classical 802.15.4 RF devices working at $250 \mathrm{~kb} / \mathrm{s}$. Second observation is that delay can rise up to several seconds (2s) when carrying big payload, and even more when adding hops (up to $4 \mathrm{~s}$ ). In this context, saving bits to reduce packet size is a crucial issue that header compression can address. In particular it enables to fully elide the source and destination IPv6 address in the IPv6 packet, saving 32 Bytes. This is a very significant gain over a 128 Bytes MTU link. As expected, 6LoWPAN always provide shorter latency than uncompressed IPv6. Header compression also shifts the
IPv6 fragmentation threshold for bigger payload, enabling to carry more data on a single IPv6 frame. In figure 3, for a payload of 70 bytes in a one hop configuration, 6LoWPAN HC keeps one 802.15.4 fragment. As a result, the round trip latency is around $1500 \mathrm{~ms}$ with Header compression, whereas we measure around $2900 \mathrm{~ms}$ without this mechanism. This represent a $45 \%$ latency gain. We calculated a average latency improvement of $15 \%$ in a no hop topology, and $17 \%$ in a one hop topology.

\section{INTEROPERABILITY}

The interoperability experiment has been performed during an IPSO interoperability event ${ }^{11}$ in March 2010. This was the first interoperability testbed between different RPL implementations. Eight companies took part in this event and five of them have tested their RPL implementations. We were the only one on PLC, the four others were using 802.15.4 RF nodes. These 5 companies used the same stack architecture, based on 802.15.4, IPv6 with 6LoWPAN (draft-ietf-6lowpanhc-06) and RPL (draft-ietf-roll-rpl-05), but each had its own implementation, differing in the OS and the devices used. The bridge between the different participant was made at the IP level by a router where a RPL implementation was running.

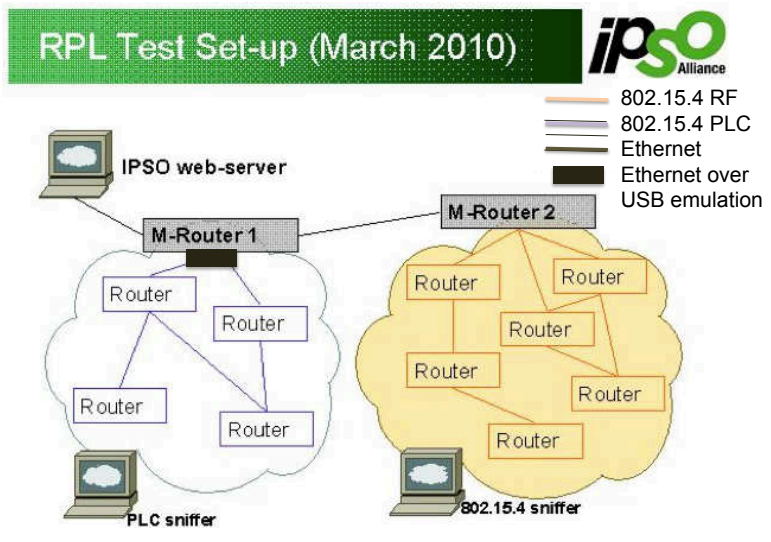

Figure 4. Interoperability test setup

As we can see on the set-up picture, the network formed was using three different media : Low-power PLC, RF (802.15.4) and Ethernet. The M-router 1 was set as the Dag root. Each router or node connected was then associated to the DAG with a classic DIS/DIO exchange. As a result, the M-router 2 associated as a node, and our PLC nodes associated to this router. The RF nodes attached directly to the M-router 1 . The Dag root has a aaaa::1 address and all the nodes (RF and PLC) have a unique global address based on their EUI-64 with a aaaa::/64 prefix.

After DAG settings, we were able to ping the node from each participating company with their global addresses from our PLC nodes. The others participants were also able to ping our node with the use of their global addresses.

\footnotetext{
${ }^{11} \mathrm{http} / / /$ www.ipso-alliance.org
} 
We also performed successfully a global repair, where the DAG root increments its instance ID and every node from the DAG has to adopt this new instance ID. This testbed showed that our stack was able to easily take part of a heterogeneous network, in a LLNs context with the use of open standard protocols.

\section{CONCLUSION AND FUture WORK}

Because our work relies on protocols that are currently drafts and not RFCs (6LoWPAN HC06 and RPL-05), trivial future work will include updates from these drafts until they become becomes RFCs, especially the RPL draft. This draft still need further revisions for improvements in the DAO mechanism and the security part. As described in the draft, RPL only defined a core set of functionalities and is not related to any particular technology. This is a strong point for the flexibility, but this also means that it let a part of the work to the implementation. The first experiment presented in this paper stands as a first interoperability testbed that is very hopeful, but it needs further work to be optimized on our network. Work still needs to be done, to tune the RPL protocol in a way that fulfill more restrictive requirements, without overpassing our node capacity. The Objective Function (OF) mechanism is also a very appreciable mechanism in RPL that we need to consider in our future work, to create a specific objective related to our PLC network, relying on low layers informations. A first set of metrics is proposed in the draft-ietf-roll-routing-metrics document. The results of the first interoperability event with RPL that we presented brings a good feedback about the capabilities of this protocol, at least on small networks. We now need further experimentation to push the capabilities of each implementation with more complex functionalities. The next testbed will certainly include more companies and more diversity, which will enhance the interoperability aspect. Another part of our future work will be to test our stack on real applications, to measure the efficiency of the implementation, in comparison with the nonstandardized protocol currently deployed. Finally, we plan to implement our stack and the specific $\mathrm{WPC}^{\mathrm{TM}}$ transceiver in a simulator, to focus on specific points that could improve the stack. We will test the performance of the stack with a homogeneous/heterogeneous dense network, and measure how far we can go on the scalability side with our actual implementation.

With this work, it is now possible to create an heterogeneous PLC/RF network in a LLNs context with the use of open standard protocol. More than dealing with coexistence, we know enable cooperation between these mediums, which is a major improvement. We have presented all the protocols of the communication stack from the physical to the network layer, including routing, to achieve the implementation of a communication stack over PLC for multi-physical layer IPv6 Networking. We have justified the need of these protocols for our LR-PAN context on powerline communication. Our experiments during the interoperability event has shown that our stack was able to interoperate with RF 802.15.4 nodes, with a bridge at the IP layer, creating a multi physical medium low power personal area network.

\section{REFERENCES}

[1] IEEE, "802.15.4," IEEE Computer Society.

[2] W. Ye, J. Heidemann, and D. Estrin, "Medium access control with coordinated adaptive sleeping for wireless sensor networks," IEEE/ACM Trans. Netw., vol. 12, no. 3, 2004

[3] T. van Dam and K. Langendoen, "An adaptive energy-efficient mac protocol for wireless sensor networks," in SenSys '03: Proceedings of the 1st international conference on Embedded networked sensor systems. New York, NY, USA: ACM, 2003, pp. 171-180.

[4] I. Rhee, A. Warrier, M. Aia, J. Min, and M. L. Sichitiu, "Z-mac: a hybrid mac for wireless sensor networks," IEEE/ACM Trans. Netw., vol. 16, no. 3, 2008

[5] E. Shih, S.-H. Cho, N. Ickes, R. Min, A. Sinha, A. Wang, and A. Chandrakasan, "Physical layer driven protocol and algorithm design for energy-efficient wireless sensor networks," in MobiCom '01: Proceedings of the 7th annual international conference on Mobile computing and networking. New York, NY, USA: ACM, 2001, pp. 272-287.

[6] L. B. Saad and B. Tourancheau, "Multiple mobile sinks positioning in wireless sensor networks for buildings," in SENSORCOMM '09: Proceedings of the 2009 Third International Conference on Sensor Technologies and Applications. Washington, DC, USA: IEEE Computer Society, 2009, pp. 264-270.

[7] C. Chauvenet, B. Tourancheau, and D. Genon-Catalot, "802.15.4, a mac layer solution for plc," in AICCSA'10. ACS/IEEE, 2010.

[8] J. W. Hui, "An extended internet architecture for low-power wireless networks - design and implementation," Ph.D. dissertation, EECS Department, University of California, Berkeley, Sep 2008.

[9] A. Dunkels, T. Voigt, and J. Alonso, "Making TCP/IP Viable for Wireless Sensor Networks," in Proceedings of the First European Workshop on Wireless Sensor Networks (EWSN 2004), work-in-progress session, Berlin, Germany, Jan. 2004.

[10] J. W. Hui and D. E. Culler, "Ip is dead, long live ip for wireless sensor networks," in SenSys '08: Proceedings of the 6th ACM conference on Embedded network sensor systems. New York, NY, USA: ACM, 2008.

[11] J. Abeillé, M. Durvy, J. Hui, and S. Dawson-Haggerty, "Lightweight ipv6 stacks for smart objects: the experience of three independent and interoperable implementations," November 2008.

[12] P. Levis, E. Brewer, D. Culler, D. Gay, S. Madden, N. Patel, J. Polastre, S. Shenker, R. Szewczyk, and A. Woo, "The emergence of a networking primitive in wireless sensor networks," Commun. ACM, vol. 51, no. 7, 2008.

[13] A. Dunkels, B. Gronvall, and T. Voigt, "Contiki - a lightweight and flexible operating system for tiny networked sensors," Local Computer Networks, Annual IEEE Conference on, vol. 0, 2004. 